\title{
Gated cone-beam CT imaging of the thorax: a reconstruction study
}

\author{
Simon Rit ${ }^{a}$, David Sarrut ${ }^{b, c}$ and Serge Miguet $^{a}$ \\ ${ }^{a}$ LIRIS, Université Lumière Lyon 2, 5 avenue Pierre Mendès-France, 69676 Bron, France; \\ ${ }^{b}$ CREATIS, INSA Lyon, Bat. Blaise Pascal, 69621 Villeurbanne Cedex, France; \\ ${ }^{c}$ Radiotherapy Department, Centre Léon Bérard, 28 rue Laënnec, 69353 Lyon, France.
}

\begin{abstract}
In radiotherapy, the respiratory motion of the patient in treatment position is determined from gated cone-beam CT images. This method consists in selecting projections according to a respiratory signal for the reconstruction of a CT image of each respiratory state. This selection can be based on the amplitude or the phase of the signal. The number of selected projections also depends on the width of the gating window. The present study compares different reconstructions using a dynamic digital phantom of the thorax and a known respiratory signal. We applied both regular and irregular motions to this phantom and reconstructed the exhale state using different widths. We evaluated these reconstructions with the signal-to-noise ratio, the contrast-to-noise ratio and a blur criterion. In the case of a regular motion, there was no difference between the amplitude and the phase. The signal quality was high, even for the smallest width, and the blur increased with width. In the case of an irregular motion, the difference was noticeable. Amplitude-based reconstructions suffered from severe artifacts with the smallest width because there were respiratory cycles for which no projection was selected. This drawback is overcome by increasing the width of the gating window. Phase-based reconstructions also allowed to avoid artifacts, whatever the width. But the blur was higher, even for the smallest width applied. These results suggest that the gating process must be adjusted in order to select at least one projection per respiratory cycle. Phase gating is a robust way to achieve this goal when respiration is irregular. Amplitude gating may be more effective in terms of blur, but the width must be carefully chosen to avoid severe artifacts. Finally, we observed the potential of dynamic reconstruction by using a motion model to deform different gated CT images toward a common reference and compute the weighted mean. The resulting CT image suffered less from artifacts than each gated CT image separately even if artifacts were still visible.
\end{abstract}

Keywords: Reconstruction, Computed Tomography (CT), Phantom, Thorax, Gated, Dynamic

\section{INTRODUCTION}

In the past few years, there has been an increasing interest of the radiation oncology community in cone-beam computed tomography (CT) scanners mounted on the gantry of medical linear accelerators. ${ }^{1,2}$ This imaging device allows to acquire CT images of the patient in treatment position. Unfortunately, like for other CT scanners, the motion of the patient during acquisition causes artifacts such as blur, streaks and bands. ${ }^{3}$ The methods available to correct these artifacts can be categorized in 3 classes, depending if they use (1) no motion information, ${ }^{4-6}(2)$ only simplified motion information like a respiratory signal, in which case the method is called respiratory-correlated or gated imaging, ${ }^{7-11}$ or (3) precise motion information like a dense vector field or a B-spline model, in which case the method is called dynamic imaging. ${ }^{12-15}$

Gated cone-beam CT images of the thorax are obtained by using a respiratory signal to sort cone-beam projections depending on their position in the periodic respiratory cycle. ${ }^{7-9,11}$ This signal can be obtained in different ways, either using an external device ${ }^{7,8,10}$ or by image processing. ${ }^{9,11,16,17}$ The question of the respiratory signal has been addressed in several works ${ }^{18-20}$ but the motion is too complex to be simplified by a $1 \mathrm{D}$ signal $^{21}$ and no definitive conclusion has been drawn.

However, even if the respiratory signal is perfect, i.e. if two identical values correspond to two identical positions of the thorax and conversely, it can be used in different ways. The first choice is between the use of

Further author information: Simon Rit: E-mail: Simon.Rit@gmail.com, Telephone: (+33) (0)4 78785150 
the amplitude of the signal versus the use of the phase of the signal. Phase is linear between processed positions such as end-exhale and end-inhale. ${ }^{22-24}$ The second choice concerns the width of the gating window. To our knowledge, no study using digital data has addressed these points.

This study aimed to study the reconstruction of a CT image from a subset of the cone-beam projections selected using a respiratory signal known a priori. We proposed to use a dynamic digital phantom and simulated a set of cone-beam projections using regular and irregular signals. We then analyzed the reconstructions obtained by varying the width of the gating window, based on the amplitude or the phase of the signals. Finally, we studied the potential of dynamic reconstruction by a weighted mean of all gated CT images along the respiratory cycle and subjected to appropriate transformation.

\section{METHOD}

\subsection{Digital phantom}

The digital thorax phantom at the exhale state was a simplified version of the Forbild phantomf(figure 1). A spherical tumor having a diameter of $3 \mathrm{~cm}$ was positioned in the lower part of the right lung. An analytical transformation was applied to this phantom to obtain the inhale state. This transformation was composed of translations and changes of volume of the different geometric parts of the phantom. Intermediate states between inhale and exhale were deduced linearly with a respiratory signal between 0 and 1 .

A dense vector field representing the deformation between end-inhale and end-exhale was computed using the demons algorithm with a Gaussian regularization ${ }^{25,26}$ (figure 1).

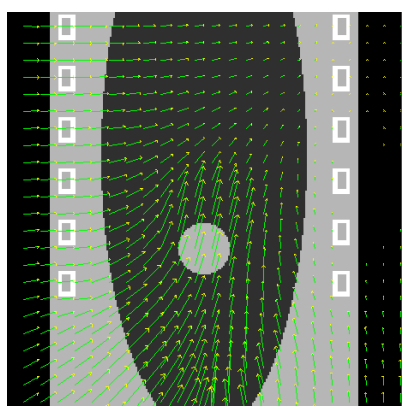

(a)

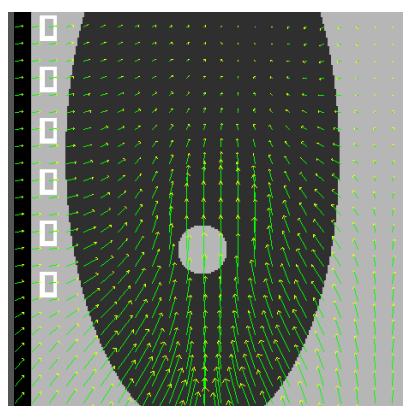

(b)

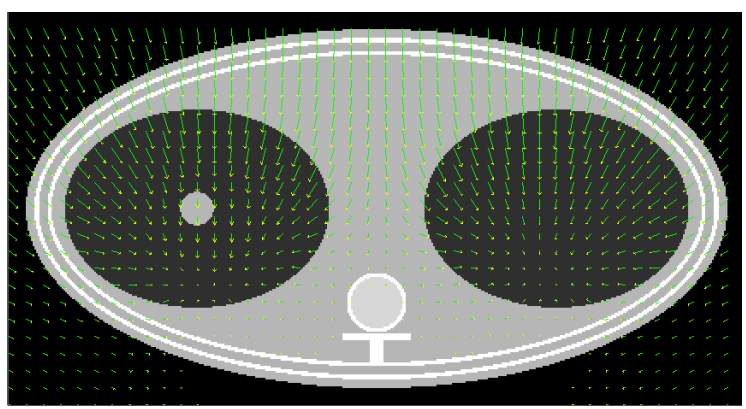

(c)

Figure 1. (a) Sagittal, (b) coronal and (c) transverse slices of the digital phantom at the inhale state with computed motion vector field.

\subsection{Respiratory signals}

Two simulated acquisitions of the dynamic phantom were obtained with two different respiratory signals (figure 2). The first regular signal was based on the asymmetric sinusoidal model $z=z_{0}+b \cos ^{2 n}(\pi t / \tau)$ with $n=2$, $\tau=4 \mathrm{~s}, z_{0}=0$ and $b=1 .{ }^{27,28}$ The second irregular signal was computed using a stochastic adaptation of the model with $n=2, \tau=4 \pm 0.5 \mathrm{~s}, z_{0}=0 \pm 0.07, b=1 \pm 0.07$. The distribution was normal for $z_{0}$ and $\log$ normal for $b$ and $\tau .{ }^{29}$ After computation, it was normalized between 0 (exhale) and 1 (inhale). The sampling period was set to 0.18 seconds. For each motion type, 640 analytical projections were computed regularly along a circular source trajectory using the open-source software $T a k e^{\dagger}$. The parameters were chosen to simulate acquisition by an Elekta Synergy system ${ }^{\mathrm{TM}}$ (Elekta Oncology Systems Ltd., Crawley, West Sussex, United Kingdom). ${ }^{1}$

\subsection{Reconstruction}

Static 3D reconstruction We implemented the method proposed by Feldkamp et al ${ }^{30}$ for cone-beam reconstruction (figure 3). The limited size of the detector provided truncated cone-beam projections and the resulting artifact was partially corrected using the heuristical method described by Ohnesorge et al. ${ }^{31}$

\footnotetext{
*http://www.imp.uni-erlangen.de/forbild/english/results/thorax/thorax.htm

${ }^{\dagger}$ http://www.cvl.isy.liu.se/Research/Tomo/take/index.html
} 


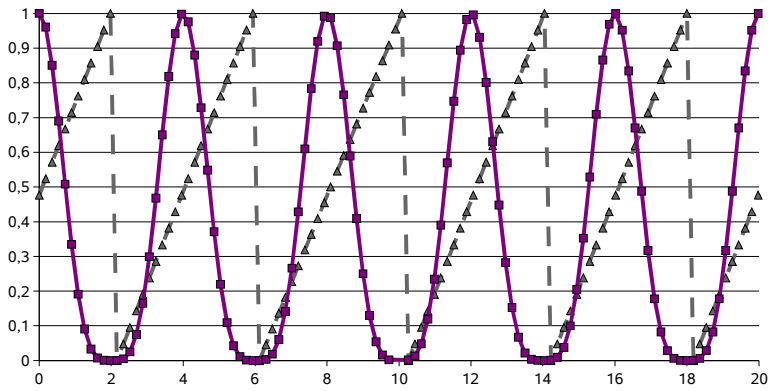

(a)

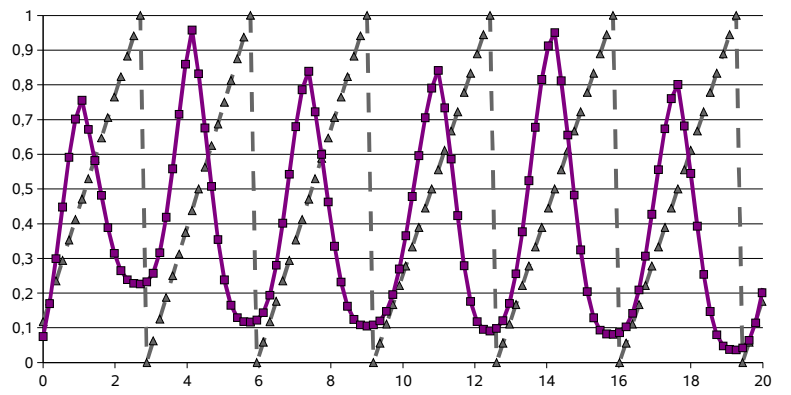

(b)

Figure 2. The first 20 seconds of the (a) regular and (b) irregular respiratory signals with their computed phase (dashed lines). Each point plotted on the curves corresponds to the acquisition of a cone-beam projection.

(1)
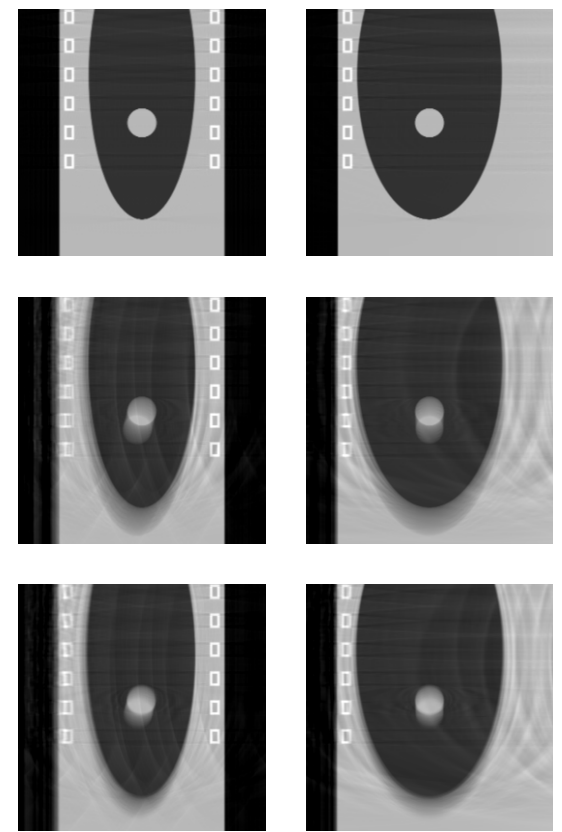

(a)

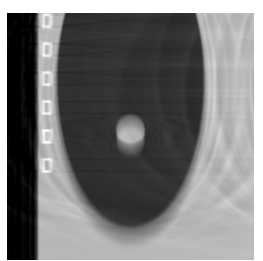

(b)
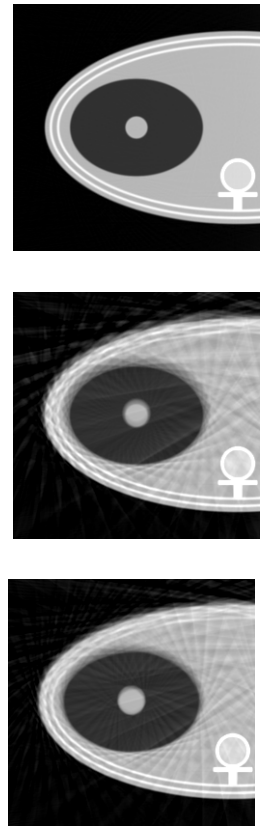

(c)

Figure 3. (a) Sagittal, (b) coronal and (c) transverse slices, of the reconstruction using the 640 projections of (1) the static phantom at end-exhale, and of the dynamic phantom with (2) a regular signal and (3) an irregular signal. The artifacts in (2) and (3) are due to the motion of the phantom between cone-beam projections. 
Gated reconstruction The gating window determines which projections are used in the reconstruction process according to the respiratory signal. We used a gating window centered on the end-exhale position with a varying width $w$. We studied two different inputs: the amplitude of the respiratory signal directly, and the phase, obtained by processing the respiratory signal. ${ }^{22,24}$ End-exhale phase was set to $0 \%$. The phase between two consecutive end-exhale phases is set linearly between $0 \%$ and $100 \%$ (figure 2 ).

Dynamic reconstruction We observed the potential of the use of a more complex motion than just a 1D signal in the reconstruction. Several studies ${ }^{14,15,32}$ have proposed to deform each backprojection of the filtered projection using a motion model. If the motion is discretized into several positions, this is equivalent to computing the weighted mean of the different gated CT images along the respiratory cycle deformed toward a common reference. The weights correspond to the number of projections used to reconstruct each CT image. We know that all the points of the phantom move in phase along a straight line, so the vector field used to deform each image is the one computed in 2.1 weighted by the mean value of the respiratory signal of the selected projections.

\subsection{Criteria}

For a quantitative analysis of the reconstructions, we used three criteria previously defined in the literature. These criteria were applied to a region of interest (ROI) containing the tumor in all positions of the thorax (figure 4).

- The signal-to-noise ratio: $\operatorname{SNR}(\mathrm{dB})=20 \log _{10} \frac{A_{\text {signal }}}{A_{\text {noise }}}$ where $A_{I}=\sqrt{\frac{1}{P} \sum_{i=1}^{P} x_{i}^{2}}$ is the root mean square of the intensity of $P$ pixels in image $I$, the signal is our reference, i.e. the computed end-exhale digital phantom, and the noise is the difference between the reconstruction to be evaluated and the signal.

- The contrast-to-noise ratio: $\mathrm{CNR}=\frac{\left|\mu_{\text {foreground }}-\mu_{\text {background }}\right|}{\sigma_{\text {background }}}$ where $\mu_{R}$ and $\sigma_{R}$ are the mean and the standard deviation of the pixel values in region R. The foreground in our ROI corresponds to the position of the tumor at end-exhale.

- The blur criterion (BC) proposed by Kriminski et al. ${ }^{8}$ This method aims to quantify the blur independently from the noise by using an appropriate energy for a binary segmentation robust to noise. The optimal energy is computed with a graph-cut and used as a blur criterion. More details can be found in the article by Kriminski et al. ${ }^{8}$

\section{RESULTS}

We reconstructed the exhale state using different widths $w$ of the gating window, in the presence of a regular or an irregular motion, and based on the amplitude or the phase of the respiratory signals (figure 44). The number $N$ of projections selected by the gating window depends on both the signal and the width.

\subsection{Regular motion}

Results were similar whether using the amplitude or the phase: the SNR, the CNR and the BC gave identical curves (figure 5). The only difference was that amplitude selected more projections than phase for small widths (figure 4 ) because more time was spent at the extreme states of the respiratory cycle. Signal quality, measured by the SNR and the CNR, slightly increased until 200-250 projections were selected and decreased afterwards. The blur was minimal for the smallest width.

\subsection{Irregular motion}

Results were different whether using amplitude or phase. When using amplitude and the smallest width $w=0.05$, no projections were selected from some respiratory cycles. This caused severe visual artifacts (figure 6), confirmed numerically by the lowest values of SNR and CNR and a disturbed BC (figure 5). Results were improved when increasing the width to $w=0.1$, a value for which the blur was comparable to the one obtained with a regular motion. No such artifact was found when using the phase, whatever the width, but the minimal blur was higher than the one obtained using amplitude. 


\begin{tabular}{|c|c|c|c|c|c|c|c|c|c|c|c|c|}
\hline & $w$ & 0.05 & 0.1 & 0.2 & 0.3 & 0.4 & 0.5 & 0.6 & 0.7 & 0.8 & 0.9 & 1.0 \\
\hline \multirow{4}{*}{ 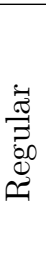 } & $N$ & 203 & 248 & 300 & 345 & 377 & 408 & 440 & 472 & 503 & 548 & 640 \\
\hline & Amplitude & & & & & & & & & & & \\
\hline & $N$ & 58 & 116 & 174 & 232 & 290 & 348 & 405 & 462 & 519 & 588 & 640 \\
\hline & Phase & & & & & & & & & & & \\
\hline \multirow{4}{*}{ 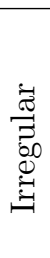 } & $N$ & 32 & 104 & 247 & 324 & 378 & 429 & 579 & 527 & 586 & 625 & 640 \\
\hline & Amplitude & & & & & & & & & & & \\
\hline & $N$ & 68 & 89 & 144 & 212 & 280 & 346 & 402 & 470 & 522 & 590 & 640 \\
\hline & Phase & & & & & & & & & & & \\
\hline
\end{tabular}

Figure 4. Coronal slices of amplitude-based and phase-based reconstructed images in the region of interest using different gating window widths $w$, resulting in the selection of a number $N$ of projections, in the presence of a regular or an irregular motion.
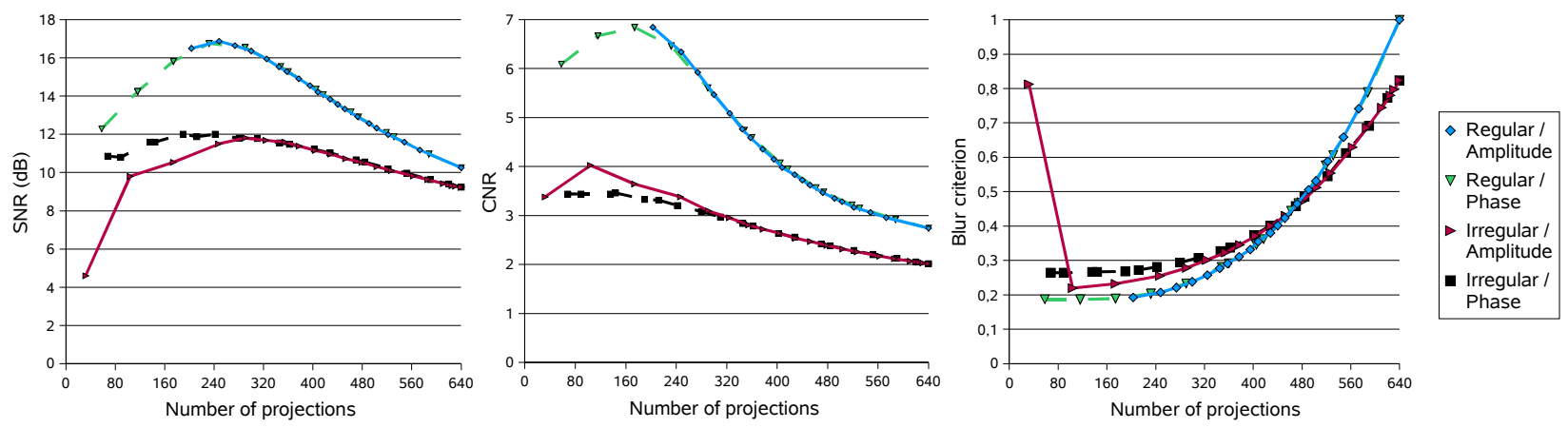

Figure 5. Signal-to-noise ratio (SNR), Contrast-to-noise ratio (CNR) and normalized blur criterion of the gated reconstruction in the region of interest (figure 4) for regular (light green and blue) and irregular (dark black and red) motions based on the amplitude (full lines) or the phase (dashed lines) of the respiratory signal. 
(1)
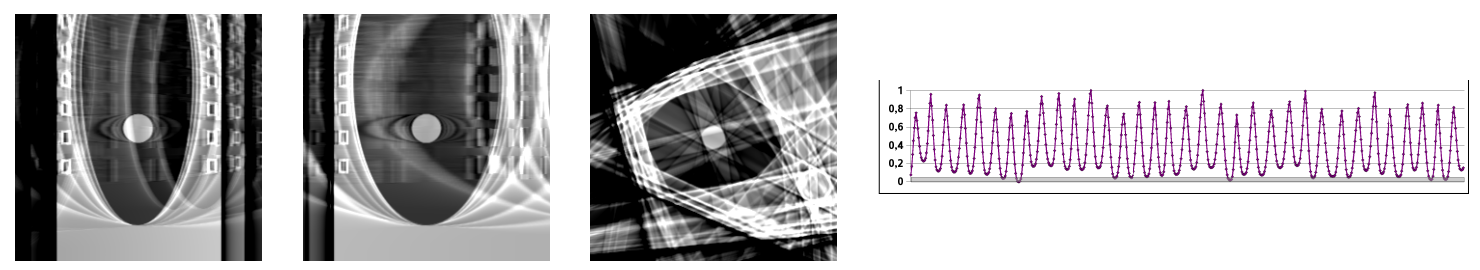

(2)

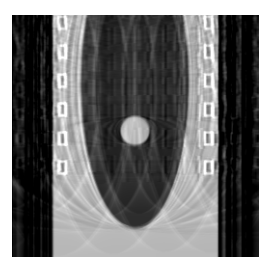

(a)

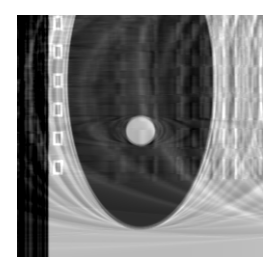

(b)

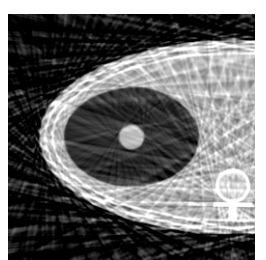

(c)

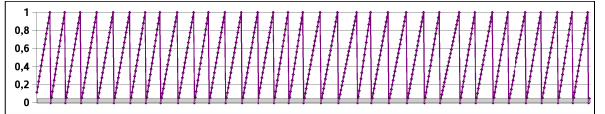

(d)

Figure 6. (a) Sagittal, (b) coronal and (c) transverse slices of (1) amplitude-based and (2) phase-based reconstructions of the phantom using an irregular motion and a gating window width $w=0.05$. Curves (d) represent the (1) amplitude and (2) phase of the respiratory signal. Gray areas at the bottom of the curves correspond to the gating windows and show the position of the selected projections. Severe artifacts are seen in the amplitude-based reconstruction because no projections were selected for some respiratory cycles, i.e. angular portions, which was not the case in the phase-based reconstruction.

\subsection{Dynamic reconstruction from gated reconstructions}

We reconstructed 10 gated CT images obtained with the same window width $w=0.1$ and different window centers $c$ in order to select all the projections once, and only once, in all gated reconstructions. This window was applied to the amplitude of the signal to minimize the blur. Table 1 summarizes the number of projections selected for the different motions and the different reconstructions. Figure 7 compares the resulting images with other reconstructions. Figure 8 is a quantitative comparison of the different reconstructions.

\begin{tabular}{|c|c|c|c|c|c|c|c|c|c|c|}
\hline$c$ & 0.05 & 0.15 & 0.25 & 0.35 & 0.45 & 0.55 & 0.65 & 0.75 & 0.85 & 0.95 \\
\hline Regular motion & 248 & 52 & 45 & 32 & 31 & 32 & 32 & 31 & 45 & 92 \\
\hline Irregular motion & 104 & 143 & 77 & 54 & 51 & 50 & 48 & 59 & 39 & 15 \\
\hline
\end{tabular}

Table 1. Number of projections selected for a gating window width $w=0.1$ and different window centers $c$, depending on the motion type. The sum of each line is equal to the total number of projections (640), because gating windows are strictly adjacent between 0 and 1 .

Dynamic reconstruction significantly improves the quality of the image. SNR and CNR values are close to the reference, i.e. reconstruction with the same number of projections (640) but without motion (figure 8). The blur remains minimal, as in gated CT reconstruction. However, visually, the streak artifacts are not fully eliminated but only attenuated (figure 7).

\section{DISCUSSION}

This study of a numerical and dynamic phantom with controllable respiratory signal allows to observe the reconstruction obtained with different widths of the gating window, and variations between the amplitude or the phase of the respiratory signal.

In the presence of a regular motion, amplitude and phase give similar results (figure 5). Minimal width applied to amplitude is a good compromise between the signal quality of the reconstruction and the blur due to the motion. We conclude like Dietrich et $\mathrm{al}^{7}$ that increasing the width does not significantly increase the signal quality because the additional projections selected are angularly adjacent to the others and, as a consequence, do not fill regularly the angular gaps along the source trajectory. 


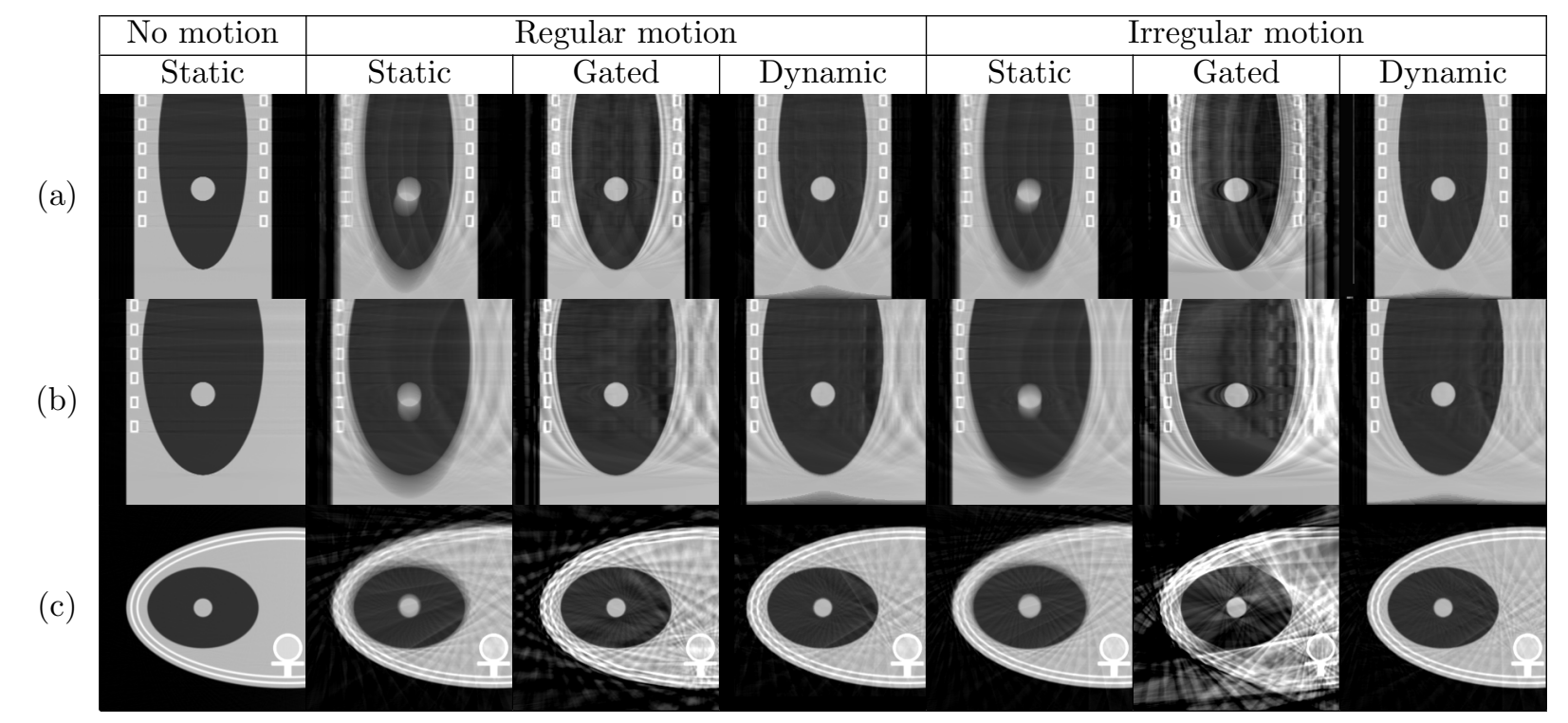

Figure 7. (a) Sagittal, (b) coronal and (c) transverse slices of the reconstructed images with static reconstruction, gated reconstruction or dynamic reconstruction, without motion or in the presence of regular motion or irregular motion.

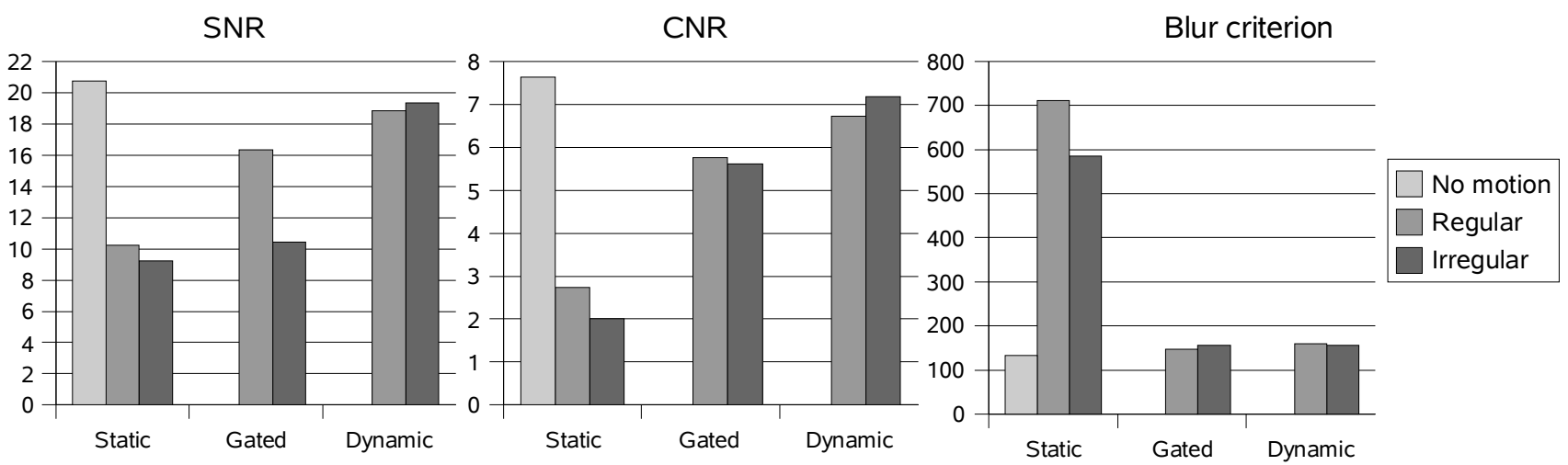

Figure 8. Quantitative criterion applied to the reconstructions displayed in figure 7 . 
Things are slightly more complicated when the phantom is subjected to irregular motion. The use of minimal width applied to the amplitude of the respiratory signal causes severe artifacts (figure 6). There are two strategies to overcome this problem. The first one is intuitive and consists in increasing the width of the gating window until reaching a good compromise between signal quality and blur due to the motion. But this can be difficult to achieve when using real data. The second strategy is to use the phase of the respiratory signal which equates to deducing a regular signal from the irregular one. This is efficient in terms of signal quality of the reconstructed image because projections are more regularly selected along the source trajectory (figure 5). But, because of the irregular nature of the motion, the reconstruction is based on projections corresponding to different positions of the thorax, which causes blur (figure 5).

The quantitative comparison of the reconstructions obtained with regular and irregular motions is not possible in this case. The observation of the respiratory signals (figure 2) and the number of projections selected for a given width of the gating window (figure 4) and for different motion patterns demonstrate that a lot more projections are available near the exhale position in the case of a regular signal than in the case of an irregular signal. It remains desirable, in radiotherapy, to be able to reconstruct the extreme position occupied by the tumor in both cases, even if this is less likely achieved with an irregular signal than a regular signal. A similar study conducted on an intermediate position in the respiratory cycle would provide interesting complementary results.

If the motion is fully known, one can deform the different gated reconstructions toward a same reference image, calculate the weighted mean and thus obtain clearly better results (figures 7 and 8). Streak artifacts are nevertheless still visible. Dynamic reconstruction assumes that the deformation of each backprojection should conduct to the same result as the reconstruction without motion. This heuristic is false because the analytic formulation of the reconstruction assumes that the integral along the ray follows a straight line. There is yet no analytic reconstruction formula for any complex deformation. Moreover, we simplified the motion model of the respiration by discretizing it in only ten different positions.

\section{CONCLUSION}

This study points out the pros and cons of amplitude-based and phase-based reconstructions for gated cone-beam imaging of the thorax. It shows the difficulty of finding a compromise between signal quality and blur. Dynamic reconstruction is potentially very powerful but the achievement of the motion model is still an issue. Future works should address the optimization of acquisition parameters for gated imaging, such as gantry speed, the study of other reconstruction algorithms, particularly for dynamic reconstruction, and a similar comparative study using real data.

\section{ACKNOWLEDGMENTS}

This work was supported in part by Elekta Oncology Systems.

\section{REFERENCES}

1. D. Jaffray and J. Siewerdsen, "Cone-beam computed tomography with a flat-panel imager: initial performance characterization," Medical Physics 27(6), pp. 1209-1343, 2000.

2. D. Jaffray, J. Siewerdsen, J. Wong, and A. Martinez, "Flat-panel cone-beam computed tomography for image-guided radiation therapy," International Journal of Radiation Oncology Biology Physics 53(5), pp. 1337-1349, 2002.

3. C. Ritchie, J. Godwin, C. Crawford, W. Stanford, H. Anno, and Y. Kim, "Minimum scan speeds for suppression of motion artifacts in CT," Radiology 185(1), pp. 37-42, 1992.

4. A. Dhanantwari, S. Stergiopoulos, and I. Iakovidis, "Correcting organ motion artifacts in X-ray CT medical imaging systems by adaptive processing. I. Theory," Medical Physics 28(8), pp. 1562-1576, 2001.

5. O. Helenon, D. Chanin, M. Laval-Jeantet, and J. Frija, "Artifacts on lung CT scans: removal with Fourier filtration," Radiology 171, pp. 572-574, 1989.

6. L. Spies and R. Luhta, "Characterization and correction of temporal artifacts in CT," Medical Physics 32(7), pp. 2222-2230, 2005. 
7. L. Dietrich, S. Jetter, T. Tücking, S. Nill, and U. Oelfke, "Linac-integrated 4D cone beam CT: first experimental results," Physics in Medicine and Biology 51(11), pp. 2939-2952, 2006.

8. S. Kriminski, M. Mitschke, S. Sorensen, N. Wink, P. Chow, S. Tenn, and T. Solberg, "Respiratory correlated cone-beam computed tomography on an isocentric C-arm," Physics in Medicine and Biology 50(22), pp. 5263-5280, 2005.

9. T. Li, L. Xing, P. Munro, C. McGuinness, M. Chao, Y. Yang, B. Loo, and A. Koong, "Four-dimensional cone-beam computed tomography using an on-board imager," Medical Physics 33(10), pp. 3825-3833, 2006.

10. J. Sillanpaa, J. Chang, G. Mageras, H. Riem, E. Ford, D. Todor, C. Ling, and H. Amols, "Developments in megavoltage cone beam CT with an amorphous silicon EPID: reduction of exposure and synchronization with respiratory gating," Medical Physics 32(3), pp. 819-829, 2005.

11. J.-J. Sonke, L. Zijp, P. Remeijer, and M. Van Herk, "Respiratory correlated cone beam CT," Medical Physics 32(4), pp. 1176-1186, 2005.

12. C. Blondel, G. Malandain, R. Vaillant, and N. Ayache, "Reconstruction of coronary arteries from a single rotational X-ray projection sequence," IEEE Transactions on Medical Imaging 25(5), pp. 653-663, 2006.

13. S. Bonnet, A. Koenig, S. Roux, P. Hugonnard, R. Guillemaud, and P. Grangeat, "Dynamic X-ray computed tomography," Proceedings of the IEEE 91(10), pp. 1574-1587, 2003.

14. P. Grangeat, A. Koenig, T. Rodet, and S. Bonnet, "Theoretical framework for a dynamic cone-beam reconstruction algorithm based on a dynamic particle model," Physics in Medicine and Biology 47(15), pp. 2611$2625,2002$.

15. T. Li, E. Schreibmann, Y. Yang, and L. Xing, "Motion correction for improved target localization with on-board cone-beam computed tomography," Physics in Medicine and Biology 51(10), pp. 253-267, 2006.

16. S. Rit, D. Sarrut, and C. Ginestet, "Respiratory signal extraction for 4D CT imaging of the thorax from conebeam CT projections," in Medical Image Computing and Computer-Assisted Intervention (MICCAI2005), 2005.

17. L. Zijp, J.-J. Sonke, and M. Van Herk, "Extraction of the respiratory signal from sequential thorax conebeam X-ray images," in International Conference on the Use of Computers in Radiation Therapy, 2004.

18. X. Allen Li, C. Stepaniak, and E. Gore, "Technical and dosimetric aspects of respiratory gating using a pressure-sensor motion monitoring system," Medical Physics 33(1), pp. 145-154, 2006.

19. N. Koch, H. H. Liu, G. Starkschall, M. Jacobson, K. Forster, Z. Liao, R. Komaki, and C. Stevens, "Evaluation of internal lung motion for respiratory-gated radiotherapy using MRI: part I-correlating internal lung motion with skin fiducial motion," International Journal of Radiation Oncology Biology Physics 60(5), p. 1459, 2004.

20. L. Simon, P. Giraud, V. Servois, and J.-C. Rosenwald, "Lung volume assessment for a cross-comparison of two breathing-adapted techniques in radiotherapy," International Journal of Radiation Oncology Biology Physics 63(2), pp. 602-609, 2005.

21. J. Blackall, S. Ahmad, M. Miquel, J. McClelland, D. Landau, and D. Hawkes, "MRI-based measurements of respiratory motion variability and assessment of imaging strategies for radiotherapy planning," Physics in Medicine and Biology 51(17), pp. 4147-4169, 2006.

22. T. Kleshneva, J. Muzik, and M. Alber, "An algorithm for automatic determination of the respiratory phases in four-dimensional computed tomography," Physics in Medicine and Biology 51(16), pp. N269-N276, 2006.

23. W. Lu, P. Parikh, J. Hubenschmidt, J. Bradley, and D. Low, "A comparison between amplitude sorting and phase-angle sorting using external respiratory measurement for 4D CT," Medical Physics 33(8), pp. 29642974, 2006.

24. N. Wink, C. Panknin, and T. Solberg, "Phase versus amplitude sorting of 4D-CT data," Journal of Applied Clinical Medical Physics 7(1), pp. 77-85, 2005.

25. V. Boldea, D. Sarrut, and C. Carrie, "Comparison of 3D dense deformable registration methods for breathhold reproducibility study in radiotherapy," in SPIE Medical Imaging: Visualization, Image-Guided Procedures, and Display, 5747, 2005.

26. D. Sarrut, V. Boldea, M. Ayadi, J.-N. Badel, C. Ginestet, and S. Clippe, "Non-rigid registration method to assess reproducibility of breath-holding with $\mathrm{ABC}$ in lung cancer," International Journal of Radiation Oncology Biology Physics 61(2), pp. 281-294, 2005. 
27. A. Lujan, E. Larsen, J. Balter, and R. Ten Haken, "A method for incorporating organ motion due to breathing into 3D dose calculations," Medical Physics 26(5), pp. 715-720, 1999.

28. Y. Seppenwoolde, H. Shirato, K. Kitamura, S. Shimizu, M. Van Herk, J. Lebesque, and K. Miyasaka, "Precise and real-time measurement of 3D tumor motion in lung due to breathing and heartbeat, measured during radiotherapy," International Journal of Radiation Oncology Biology Physics 53(4), pp. 822-834, 2002.

29. R. George, S. Vedam, T. Chung, V. Ramakrishnan, and P. Keall, "The application of the sinusoidal model to lung cancer patient respiratory motion," Medical Physics 32(9), pp. 2850-2861, 2005.

30. L. Feldkamp, L. Davis, and J. Kress, "Practical cone-beam algorithm," Journal of Optical Society of America A 1(6), pp. 612-619, 1984.

31. B. Ohnesorge, T. Flohr, K. Schwarz, J. Heiken, and K. Bae, "Efficient correction for CT image artifacts caused by objects extending outside the scan field of view," Medical Physics 27(1), pp. 39-46, 2000.

32. C. Ritchie, C. Crawford, J. Godwin, K. King, and Y. Kim, "Correction of computed tomography motion artifacts using pixel-specific back-projection," IEEE Transactions on Medical Imaging 15(3), pp. 333-342, 1996. 